\title{
Structure of NiCrAIY Coatings Deposited on Oriented Single Crystal Superalloy Substrates by Laser Cladding
}

\author{
Rui Vilar ${ }^{1, a}$, Edson Costa Santos ${ }^{2, b}$ \\ ${ }^{1}$ Technical University of Lisbon, IST, Department of Material Science, Rua Rovisco Pais, 1000-049, \\ Lisbon, Portugal \\ ${ }^{2}$ Kyushu University, Department of Mechanical Engineering, Motooka 744, Nishi-ku, 819-0395, \\ Fukuoka, Japan \\ arui.vilar@ist.utl.pt, bsantos@mech.kyushu-u.ac.jp
}

Key words: Single-crystal turbine blades; laser deposition; nickel superalloys; coating; solidification microstructure.

\begin{abstract}
In the present work single and multiple layer NiCrAlY coatings were produced by laser cladding on (100) single-crystalline substrates of SRR99 Ni-based superalloy. Detailed structural characterisation and texture analysis by optical microscopy, scanning electron microscopy, X-ray diffraction and Rutherford backscattering showed that the NiCrAlY coatings consisted essentially of gamma phase with yttrium oxide (Y2O3) and a small proportion of yttrium-aluminium garnet (A15Y3O12) precipitated in the interdendritic regions. The coatings presented a columnar dendritic structure grown by epitaxial solidification on the substrate and inherited the single-crystalline nature and the orientation of the substrate. The coating material also showed a mosaicity and a defect density similar to those of the substrate. It can be expected that the protective effect of these coatings against oxidation is greatly enhanced compared with polycrystalline coatings because high diffusivity paths, such as grain boundaries, are eliminated in single-crystalline coatings, thus reducing mass transport through the coating.
\end{abstract}

\section{Introduction}

The use of single-crystalline Ni-based superalloys represented a major advance in improving the high-temperature mechanical behaviour and increasing the service life of blades and other components in the hot section of gas turbine engines [1]. This improvement is mainly due to the elimination of grain boundaries [2] and to the alignment of the minimum-Young's-modulus direction (the $<001>$ direction) along the turbine blade axis [3], which leads to higher creep resistance and thermal fatigue respectively. However, despite the outstanding high temperature mechanical properties of these alloys, their resistance to oxidation is insufficient for many applications and they are usually coated with MCrAlX-type alloys $(\mathrm{M}=\mathrm{Fe}, \mathrm{Co}, \mathrm{Ni}, \mathrm{X}=\mathrm{Y}, \mathrm{Hf}, \mathrm{Ce}, \mathrm{Zr})[4,5]$, which form an adherent and spalling-resistant surface $\mathrm{Al}_{2} \mathrm{O}_{3}$ layer that isolates the material from the aggressive action of the environment [6]. These coatings can be applied by a wide range of techniques, such as vacuum plasma spraying [7], high-velocity oxygen-fuel (HVOF), thermal spraying [8], and laser cladding [9]. The coatings produced by laser cladding are typically free of pores and cracks [10] and their oxidation resistance is usually better than the oxidation resistance of similar coatings produced by alternative methods, due to the finer dispersion of the rare-earth-rich phases [11]. Single crystalline coatings should further improve oxidation resistance because high diffusivity paths along grain boundaries would be eliminated. The possibility of producing single crystalline NiCrAlY coatings using a laser cladding technique is investigated in the present paper.

Gaümann et al. [12,13] showed that clad layers of CMSX-4 Ni-based superalloy deposited by laser cladding on a (100) single crystalline substrate of the same material are single crystalline, with a crystallographic orientation similar to that of the substrate. This is because in laser cladding solidification starts by epitaxial growth on the substrate and the solidification structure is dendritic $[12,13]$. In cubic metals, dendrites grow preferentially along $<100>$ directions in order to minimise their surface [14] and strain energy [3,15]. Since a (100) substrate was used, the microstructure of the material consists of parallel dendrites with the same crystallographic orientation as the substrate, forming an imperfect single crystal. According to Gaümann et al. [16], the main limit to achieving a 
single crystalline structure in the deposited material is the possibility of a columnar to equiaxed solidification transition occurring when the solidification front approaches the surface, due to the progressive decrease in the temperature gradient in the liquid in front of the interfaces leading to the formation of a large constitutionally undercooled liquid layer ahead of the S/L interfaces which favours equiaxed solidification. As a result of this transition, randomly-oriented equiaxed crystals appear at the top of the deposited tracks. According to Gaümann et al. [16] and Mokadem et al. [17], epitaxy loss tends to occur close to growth direction transition zones, where a new $<100>$ dendrite growth direction is selected. The greater the angle between the temperature gradient and the dendrite growth direction, the higher the risk of equiaxed solidification. The present paper focuses on the microstructure and crystalline structure of single and multiple layers NiCrAlY coatings deposited by laser cladding on single crystalline turbine blades. The experimental conditions to produce epitaxial NiCrAlY coatings with a single crystalline structure were investigated.

\section{Experimental procedures}

Laser cladding was performed on substrates cut from a single crystalline turbine blade of SRR99 Ni-based superalloy with chemical composition Ni-4.8Co-8.5Cr-5.3Al-2.65Ta-2.05Ti-9.25W-0.5Mo (wt.\%). The blade, which had its longitudinal direction approximately parallel to the [001] crystallographic direction, was cross-sectioned perpendicularly to its axis in order to obtain substrates with surfaces nominally parallel to the (001) plane. Prior to the cladding experiments the substrates were blasted with glass microspheres to enhance absorption of laser radiation. Laser cladding was performed by the blown powder technique using a NiCrAlY-type alloy with chemical composition Ni-24.6Cr-5.72Al-0.84Y (wt.\%) as the coating material. The powder, transported by an argon jet, was delivered to the laser generated melt pool by a $3 \mathrm{~kW} \mathrm{CO}_{2}$ laser through a $2 \mathrm{~mm}$ diameter nozzle, at an angle of $45^{\circ}$ to the horizontal. The samples were moved under the stationary laser beam at constant speed, using a numerically controlled X-Y table. The laser processing parameters used were: laser beam power $=1.8 \mathrm{~kW}$, laser spot size at the surface $=2 \mathrm{~mm}$, powder feed rate $=2.4 \times 10^{-4} \mathrm{~kg} / \mathrm{s}$ and scanning velocity $=0.01 \mathrm{~m} / \mathrm{s}$. The cladding tracks were deposited in random directions. In order to achieve different coating thicknesses and to study the influence of multiple layer overlapping on dendrite orientation, samples with 1, 2 and 6 deposited layers were produced.

Transverse and longitudinal cross-sections of the coatings were prepared for structural characterization. The microstructure was studied by optical and scanning electron microscopy after electrochemical etching using a solution of $10 \mathrm{mg} \mathrm{CrO}_{3}$ in $100 \mathrm{ml}$ water and $1.5 \mathrm{~V}$. The crystallographic structure of the substrate and the coating was studied by X-ray diffraction (XRD). X-ray crystallographic texture analysis was performed on the transverse cross-section of a six-layer coating on areas on the substrate, close to the substrate-coating interface, at half-thickness, and close to the surface of the coating, using a precision two-axis goniometer. The experiments were performed using an X-ray beam spot size at the surface of the sample of $1 \mathrm{~mm}^{2}$. The $\psi$ angle was varied in the range $0-60^{\circ}$ in steps of $5^{\circ}$, while the $\varphi$ angle varied independently in the range $0-360^{\circ}$ in $5^{\circ}$ steps. Rutherford backscattering (RBS) and channelling effect (RBS-C) experiments were performed on longitudinal cross-sections of a 6-layer coating, using a Van de Graaf accelerator with a two-axis goniometer and ${ }^{4} \mathrm{He}^{+}$ions at $1.6 \mathrm{MeV}$.

\section{Results and discussions}

The orientation of the substrate and the angle between the clad track direction and the $<100>$ family directions of the substrate was determined using the high-resolution multiaxis diffractometer. The substrate orientation was obtained by finding the $\Phi$ and $\psi$ angles for both the (002) and (220) planes reflections [18]. This was achieved by varying the $\Phi$ axis and collecting the $2 \theta$ Bragg position for a given $\omega$ range $(\omega=\theta \pm \psi)$. Stereographic projections of the substrate were constructed using CaRIne Crystallography 3.1 software. The normal to the substrate surface, as well as the wall longitudinal mid-plane, are at $10.6^{\circ}$ from the [001] direction, in disagreement with the nominal orientation assumed for the substrate, which was $Z / /[001]$. Taking this into consideration, the surface of the substrate used is approximately parallel to the (530) crystallographic plane of the material. 
Micrographs of the interface between the substrate and the first clad layer are shown in Fig. 1a. Near the substrate, the clad presents a featureless zone formed by planar solid-liquid interface solidification (region 1), followed by a thin transition layer with a cellular structure (region 2), and a dendritic region that occupies most of the clad thickness (region 3). The dendrites do not present well-developed secondary or higher-order arms (cellular dendrites, [19]). The mean primary arm interdendritic distance is about $5 \mu \mathrm{m}$. Families of dendrites with slightly different orientations are observed in the coating near the substrate/coating interface (fig. 1b), but after a certain growth distance dendrites with the main stems parallel to a direction about $10^{\circ}$ to the normal to the interface clearly predominate and the microstructure consists of an array of long and parallel dendrites. Families of misoriented dendrites may grow from regions of the substrate with different orientations, due to substrate mosaicity, as show in Fig. 1b, where several subgrains are visible in the clad, each consisting of parallel columnar dendrites growing in slightly different directions. An important other source of misoriented dendrites is the $\mathrm{Ni} / \mathrm{Ni}_{3} \mathrm{Al}$ eutectic cells existing in the substrate. The eutectic has a lower melting point than the solid solution and no preferred orientation. When eutectic cells are melted by the laser beam, the solid/liquid interface is distorted and dendrites growing from the eutectic are strongly misaligned in relation to the average orientation of neighbouring columnar dendrites grown directly from axially-oriented substrate dendrites. (Fig. 1c).

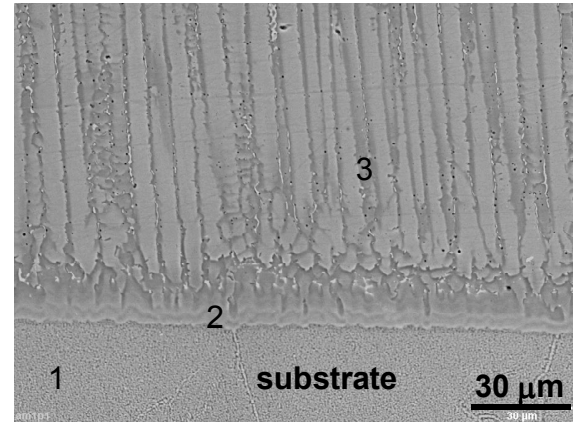

(a)

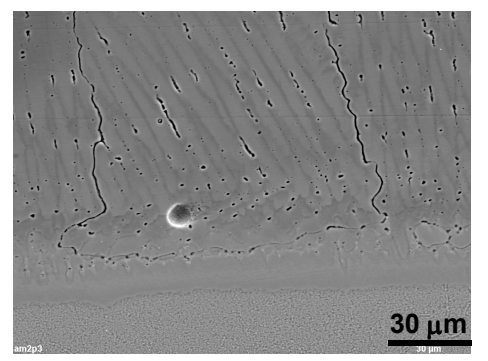

(b)

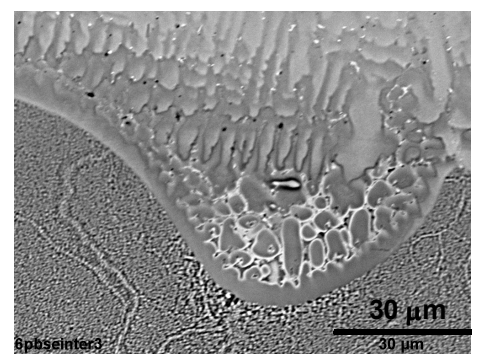

(c)

Figure 1. SEM micrographs of the transverse cross-section of a single layer coating: (a) substrate coating interface (b) families of dendrites with slightly different primary arm orientation caused by substrate mosaicity (c) eutectic cell melted by the laser beam leading to different primary dendritic growth direction.

Precipitates were detected in the interdendritic regions in backscattered-electron images, particularly with higher magnification. X-ray energy-dispersive spectrometry (XEDS) point analysis and mapping lead to the conclusion that these precipitates consist predominantly of yttrium oxide $\left(\mathrm{Y}_{2} \mathrm{O}_{3}\right)$. Some particles are formed of two phases, probably $\mathrm{Y}_{2} \mathrm{O}_{3}$ and $\mathrm{Al}_{5} \mathrm{Y}_{3} \mathrm{O}_{12}$. By selecting appropriate deposition parameters, dilution contamination of the coating by the substrate material can be avoided. XEDS measurements of the concentration profile across the substrate/coating interface of a single layer cladding showed that the dilution region is confined to a few tens of micrometers. When new layers of material are added to previously deposited ones, solidification starts by epitaxial growth as in single-layer cladding (Fig. 2). The plane front solidification region, clearly visible in the first clad layer, is very narrow or entirely absent in the upper layers, due to a progressive decrease of the temperature gradient as new layers of material are deposited. The microstructure is similar in all layers and consists of parallel cellular dendrites with an average orientation similar to the orientation of dendrites in the previous layer. In general the dendrite stems do not show continuity from layer to layer, but dendrites preserve their orientation across consecutive layers, as observed in Fig. 2. As a result, the microstructure of a multilayer coating consists of parallel columnar dendrites with primary arms aligned perpendicularly to the substrate surface, which grow throughout the thickness of a single layer but preserve their mean orientation across all layers. In agreement with the results of X-ray diffractometry, which showed that the [001] direction is at approximately $10.6^{\circ}$ to the normal to the substrate surface, the columnar dendrites observed in Fig. 2 do not grow perpendicularly to the interface between the substrate and the coating, but are all tilted about $10^{\circ}$ in relation to it. The [100] fibre textures of the coating and of the substrate arise as a consequence of competitive growth of 
dendrites. As mentioned earlier, columnar dendrites grow far from the limit of stability of the plane front and adopt an orientation which is as close as possible to the heat flux direction, but follow one of the six $<100>$ preferred directions in cubic materials. Fortunately, in columnar constrained growth conditions, misoriented dendrites must grow at a greater solidification rate than dendrites oriented in the temperature gradient direction. As a result, their dendrite tip temperature is lower and according to Tamman and Botshwar's criterion, they tend to be eliminated in the competition with better aligned adjacent dendrites, which solidify with lower dendrite tip undercooling. The consequence of this competition is that misoriented dendrites growing, for example, from eutectic cells are suppressed after a certain growth time, thus reducing the scatter in the crystallographic orientation of the dendrites.

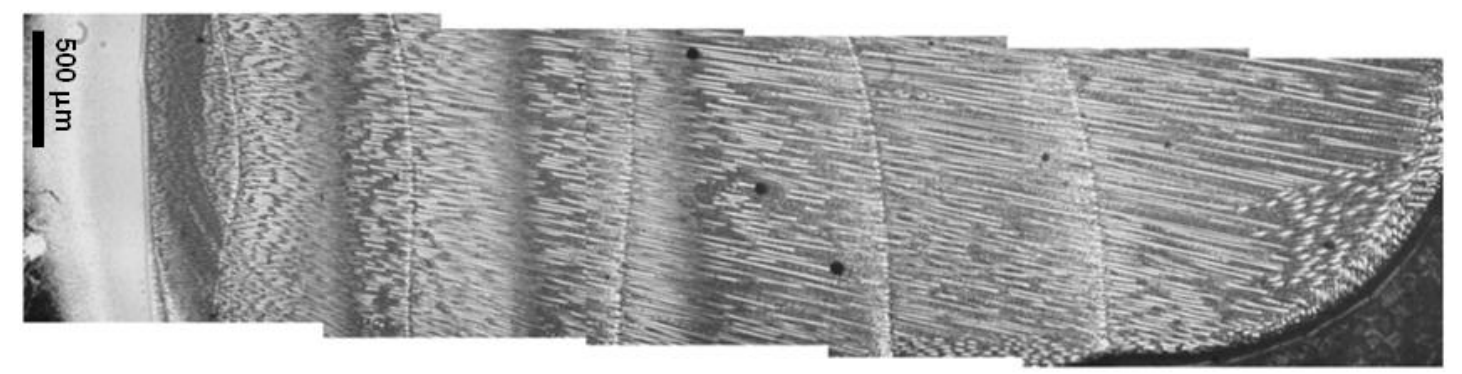

Figure 2. Microstructure in the transverse cross-section of a six-layer coating.

At the surface of the upper layer of the longitudinal cross-section, randomly-oriented equiaxed dendrites appear (Fig. 3). A close examination of the microstructure of this region suggests that they nucleate at surface impurities, such as floating oxide particles, or on powder particles impinging on the surface of the melt pool and grow by equiaxed growth into the liquid. These misoriented crystals are remelted when new layers are deposited so they do not have a negative impact on the microstructure.

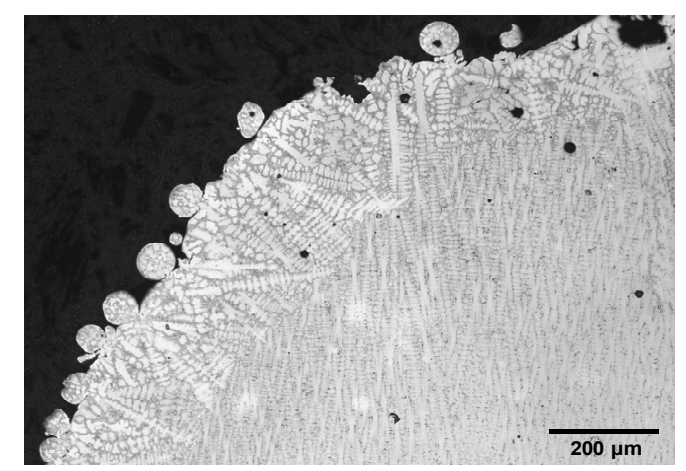

Figure 3. Optical micrograph of the longitudinal cross-section: heterogeneous nucleation at the surface leading to spurious grains at the top of the coating.

The pole figures (Fig. 4) were plotted on the transverse cross-section of a six-layer coating in positions corresponding to the substrate, interface between substrate and coating material, at half height of the coating and near the top of the coating. The (100) pole distributions (Fig. 4) are very narrow and similarly located in the substrate and throughout the coating thickness, confirming that both the coating and the substrate are strongly textured and present similar crystallographic orientations. The width of the pole distributions is narrow, typical of an imperfect single crystalline material. The pole distributions are slightly narrower in the substrate than in the coating and its width increases with increasing distance from the substrate, suggesting that the dispersion of crystallographic orientation increases as new layers of material are overlapped. In the pole figure plotted near the top of the coating (Fig. 4d), two new [100] pole families appear close to the normal of the sample surface, which certainly correspond to the stray grains observed in the upper layer of the multilayer coating shown in Fig. 3. These conclusions were confirmed by examination of the [111] pole figures. 

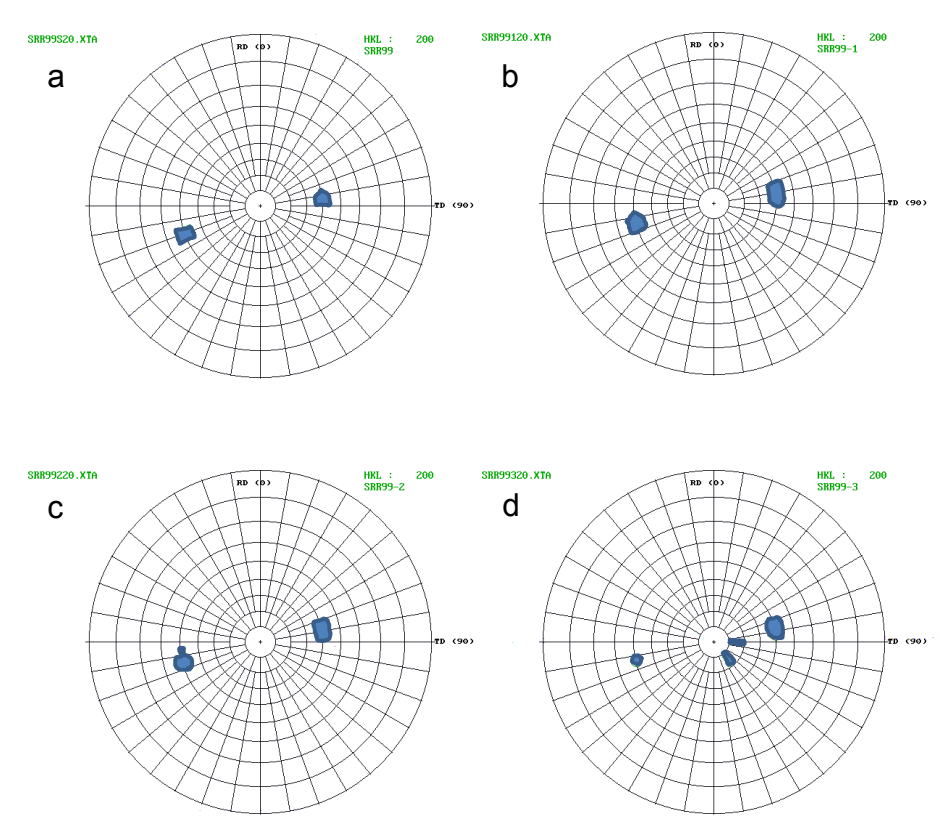

Figure 4. (100) Pole figures of the tranverse cross section: (a) substrate (S), (b) near substrate (interface, SL), (c) coating half height (Lh), and (d) coating top (Lt).

RBS-C experiments carried out on the longitudinal cross-section of the six-layer coating confirm that there is less than two degrees difference between the [022] pole orientation in the substrate and in the coating. The RBS spectra from the substrate and coating in channelling (i.e. with the beam aligned with the [022] crystalline axis) and non-channelling conditions are shown in figure 5. The backscattering yield under channelling conditions is an indication of crystalline perfection, and it was lower in the coating than in the substrate, showing that the crystalline quality of the coating is very good, at least comparable to the substrate.
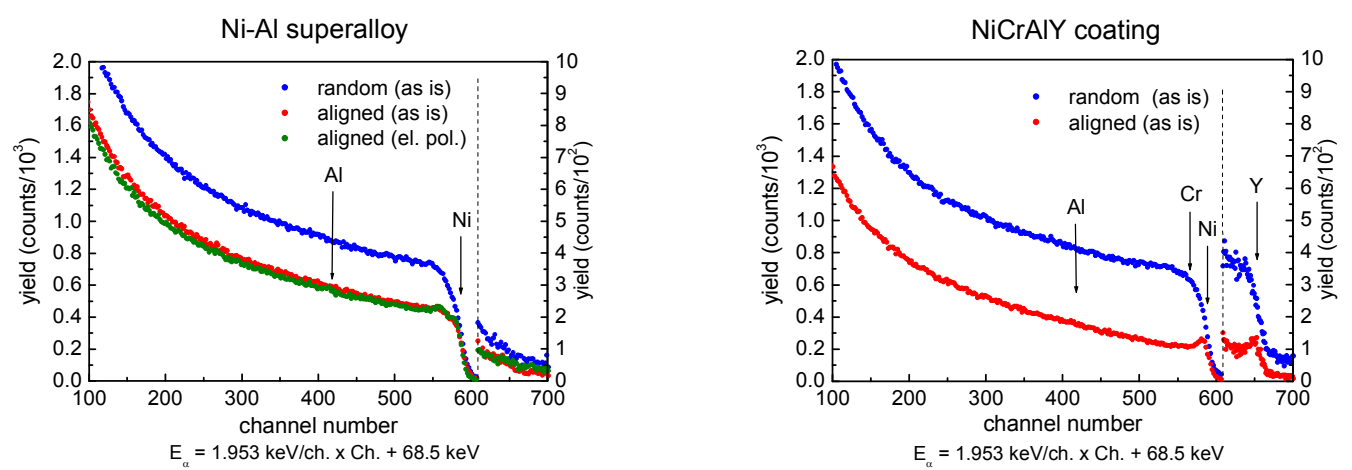

Figure 5. Random and [022] aligned Rutherford backscattering spectra of the longitudinal cross-section of the substrate (a) and the coating (b).

\section{Conclusions}

This work showed that it is possible to produce single crystalline coatings of a NiCrAlY alloy on (100) single crystalline turbine blade material by means of laser cladding. The crystalline quality of the deposited material observed by RBS appears to be better than the crystalline quality of the substrate and perfectly adequate for engineering applications, and the coatings are expected to present good properties. 


\section{References}

[1] Versnyder, F.L. and M.E. Shank, Development of Columnar Grain and Single Crystal High Temperature Materials through Directional Solidification. Materials Science and Engineering, 1970. 6(4): p. 213-247.

[2] McLean, M., Directionally Solidified Materials for High Temperature Service. 1983, London: The Metals Society.

[3] D'Souza, N., et al., Directional and single-crystal solidification of Ni-base superalloys: Part I. The role of curved isotherms on grain selection. Metallurgical and Materials Transactions A-Physical Metallurgy and Materials Science, 2000. 31(11): p. 2877-2886.

[4] Han, Y.F., Z.P. Xing, and M.C. Chaurvedi, Structural Intermetallics. The Minerals, Metals and Materials Society, ed. M.V. Nathal, et al. 1997.

[5] Reed, R.C., The Superalloys, Fundamentals and Applications. first ed. Cambridge University Press. 2006: Cambridge University Press. 372.

[6] Sprague, J.A. and J.L. Cocking, M-Cr-Al-X Coatings for Superalloys, in Advances in High Temperature Structural Materials and Protective Coatings, A.K. Koul, et al., Editors. 1994, National Research Council of Canada: Ottawa. p. 205-225.

[7] Brandl, W., et al., The oxidation behaviour of sprayed MCrAlY coatings. Surface \& Coatings Technology, 1996. 87-8(1-3): p. 41-47.

[8] Brandl, W., et al., The oxidation behaviour of HVOF thermal-sprayed MCrAlY coatings. Surface \& Coatings Technology, 1997. 94-5(1-3): p. 21-26.

[9] Damborenea, J.d. and A.J. Vazquez, Laser Cladding of High-Temperature Coatings. Journal of Materials Science, 1993. 28: p. 4775-4780.

[10] Vilar, R., Laser cladding. Journal of Laser Applications, 1999. 11(Special number: Laser Applications Around the World, Invited Reviews from Amongst The World's Leaders, Part 2): p. 64-79.

[11] Ribaudo, C.R., J. Mazumder, and D.W. Hetzner, Laser-Clad NiAlCrHf Alloys with Improved Alumina Scale Retention. Metallurgical Transactions B, 1992. 23(4): p. 513-522.

[12] Gaümann, M., et al., Epitaxial laser metal forming : analysis of microstructure formation. Mater. Sci. Eng. A, 1999. 271: p. 232-241.

[13] Gaümann, M., et al., Epitaxial Laser Metal Forming on a single Crystal Superalloy, in Laser Assisted Net shape Engineering 2, Proceedings of the LANE'97, M. Geiger and F. Vollertsen, Editors. 1997, Meisenbach: Bamberg. p. 651-657.

[14] Walton, D. and B. Chalmers, The Origin of the Preferred Orientation in the Columnar Zone of Ingots. Transactions of the American Institute of Mining and Metallurgical Engineers, 1959. 215(3): p. 447-457.

[15] Lee, D.N., et al., Factors determining crystal orientation of dendritic growth during solidification. Materials Chemistry and Physics, 1997. 47(2-3): p. 154-158.

[16] Gaümann, M., et al., Single-Crystal Laser Deposition of Superalloys: Processing-Microstructure Maps. Acta Materialia, 2001. 49: p. 1051-1062.

[17] Mokadem, S., et al. Microstructure control during single crystal welding and deposition of Ni-base superalloys. in TMS annual meeting 2004. 2004. Charlotte, USA.

[18]Franco, N., S. Pereira, and A.D. Siqueira, Absolute Scale Reciprocal Space Mapping on X-ray Difractometers Incorporating a Position Sensitive Detector: Application to III-Nitride Semiconductors. Materials Science Forum, 2004. 455: p. 132-136.

[19] Li, L. and R.A. Overfelt, Influence of directional solidification variables on the cellular and primary dendrite arm spacings of PWA1484. Journal of Materials Science, 2002. 37: p. 3521-3532. 